\title{
TGF-beta 1 Codon 10 Polymorphism is Associated with Cerebral SVD
}

\author{
Hong-miao Tao, Guo-zhong Chen, Xiao-dong Lu, Xiao-gang Hu, \\ Gan-ping Chen, Bei Shao
}

\begin{abstract}
Background: To clarify the role of inflammation in the pathogenesis of cerebral small vessel disease (SVD), we investigated whether the gene encoding transforming growth factor-beta 1(TGF-beta 1) is a risk factor for cerebral SVD as a whole, and for two different SVD subtypes. Methods: TGF- beta 1 codon10 (T+29C) genotype was determined in 441 Chinese patients (313 male and 128 female) with cerebral SVD and 450 control subjects (326 male and 124 female). Cerebral SVD patients were retrospectively classified into two groups based on neuroimaging findings: lacunar infarction group with 112 patients and ischaemic leukoaraiosis group with 329 patients. Results: Subjects carrying TT homozygote were susceptible to cerebral SVD [adjusted odds ratio $(\mathrm{OR})=1.44$, 95\% confidence interval (CI), 1.05-1.98; $\mathrm{P}=0.026]$. Further analysis of SVD subtypes revealed a moderate association with the ischaemic leukoaraiosis group [OR=1.60, 95\% CI, 1.14-2.25; $\mathrm{P}=0.007]$. Conclusions: Codon 10 of TGF-beta 1 might be a risk factor for SVD, specifically in ischaemic leukoaraiosis phenotype.
\end{abstract}

\begin{abstract}
RÉSUMÉ: Polymorphisme du codon 1 de TGF-bêta associé à la maladie des petits vaisseaux cérébraux. Contexte : Nous avons vérifié si le gène qui code le facteur de croissance transformant bêta-1 (TGF-beta 1) est un facteur de risque général de maladie des petits vaisseaux cérébraux (MPV) et de deux sous-types différents de MPV afin de préciser le rôle de l'inflammation dans la pathogenèse de la MPV. Méthodes : Le génotype du codon 10 de TGF-bêta $1(\mathrm{~T}+29 \mathrm{C})$ a été déterminé chez 441 patients chinois ( 313 hommes et 128 femmes) atteints de MPV et chez 450 sujets témoins (326 hommes et 124 femmes). Les patients atteints de MPV ont été classifiés rétrospectivement en 2 groupes, selon les constatations à la neuroimagerie : un groupe de 112 patients présentant des infarctus lacunaires et un groupe de 329 patients porteurs de leucoaraïose ischémique. Résultats : Les sujets homozygotes TT étaient prédisposés à la MPV cérébrale [rapport de cotes $(\mathrm{RC})$ ajusté $=1,44$; intervalle de confiance (IC) à $95 \%$ de 1,05 à 1,$98 ; p=0,026]$. L'analyse des sous-types de MPV a montré une association modérée au groupe de leukoaraïose ischémique [ $\mathrm{RC}=1,60 ; \mathrm{IC}$ à $95 \%$ de 1,14 à 2,$25 ; \mathrm{p}=0,007]$. Conclusions : Le codon 10 du gène TGF-bêta 1 pourrait être un facteur de risque de MPV, plus spécifiquement du phénotype de la leukoaraïdose ischémique.
\end{abstract}

Can. J. Neurol. Sci. 2011; 38: 869-873

Cerebral small vessel disease (SVD) accounts for a quarter of ischaemic stroke cases and is the most common cause of vascular dementia. ${ }^{1}$ The pathogenesis of cerebral SVD is poorly understood. Two pathological and radiological subtypes have been suggested: localised atherosclerotic disease in larger perforating arteries causing larger lacunar infarcts without leukoaraiosis, and diffuse disease in smaller arterioles causing multiple smaller lacunar infarcts with leukoaraiosis. ${ }^{2-5}$

Because evidence continues to mount suggesting important roles for inflammation in SVD, ${ }^{5-8}$ we hypothesize that functional genetic variations of the inflammation markers may play a role in the pathogenesis of SVD. Transforming growth factor (TGF)beta has three isoforms, TGF- beta 1 , TGF- beta 2 , and TGFbeta 3 . TGF-beta 1 is a pleiotropic cytokine with a central role in inflammation and the most pivotal TGF- beta isoform for the cardiovascular system. ${ }^{9}$ The TGF- beta 1 gene is located on chromosome 19q13. There are several commonly known (potentially) functional polymorphisms in this gene. The T+29C polymorphism of codon 10 (rs1800470), resulting in a leucine to proline substitution, has also been shown to be associated with the serum level of TGF- beta $1 .^{10-11}$ To test this hypothesis and provide insights into the pathogenesis of cerebral SVD or differences between phenotypes, we evaluated the association of polymorphisms of the TGF- beta 1 gene at codon $10(\mathrm{~T}+29 \mathrm{C})$ with SVD in a Chinese population.

From the School of Medicine (HMT, GZC), Jinhua College of Profession \& Technology; Department of Clinical Laboratory (XDL), Department of Radiology (XGH), Jinhua Central Hospital; Department of Neurology (GPC), Jinhua People's Hospital, Jinhua; Cerebrovascular Unit (BS), Department of Neurology, the First Affiliated Hospital, Wenzhou Medical College, Wenzhou, Zhejiang Province, the People's Republic of China.

Received April 7, 2011. Final Revisions Submitted May 19, 2011. Correspondence to: Hong-miao Tao, School of Medicine, Jinhua College of Profession \& Technology, Jinhua, 321007, Zhejiang Province, The People's Republic of China. 


\section{Materials AND Methods}

\section{Study Population}

The study protocol included history taking, neurological examination, extracranial and transcranial ultrasound, neuroimaging and laboratory testing. This study was conducted on patients who were consecutively admitted to the Department of Neurology of Jinhua Central Hospital and Jinhua People's Hospital (Jul, 2007 to Mar, 2011). Four hundred and forty-one consecutive patients presenting with SVD were recruited. All patients had brain imaging, imaging of the extracranial cerebral vessels and electroencephalogram (EEG). Where clinical suspicion was higher for a cardioembolic source, echocardiography was performed. Cerebral small vessel disease was defined as a clinical lacunar syndrome with a compatible lesion on magnetic resonance image (MRI) or computed tomogram (CT). Patients with subcortical lesion $1.5 \mathrm{~cm}$ in diameter, cortical infarct of any size, a potential cardioembolic source, and large-vessel disease defined as carotid, vertebral, or basilar intracranial artery stenosis $50 \%$ were excluded. Four hundred and fifty controls free of symptomatic cerebrovascular disease, were recruited in a routine annual health check up from the same geographic locations as the patients. Sampling was stratified to a similar distribution of age, sex and conventional vascular risk factors as the patients in the study. Subjects were excluded after performing a CT or MRI scan if was highly suspected of having a stroke. The evaluation of risk factors in both cases and controls included age, sex, body mass index $\left(\mathrm{BMI}, \mathrm{kg} / \mathrm{m}^{2}\right.$ ), hyperlipemia, smoking status, hypertension and diabetes mellitus.

Systemic arterial hypertension was defined as a systolic blood pressure of $140 \mathrm{~mm} \mathrm{Hg}$, and/or a diastolic blood pressure of 90 $\mathrm{mm} \mathrm{Hg}$, at least on two separate occasions, or antihypertensive treatment. Hyperlipemia was defined as either an elevated fasting total cholesterol level above $220 \mathrm{mg} / \mathrm{dL}$ or fasting triglyceride level above $150 \mathrm{mg} / \mathrm{dl}$ or current treatment with lipid-lowering medication. ${ }^{12}$ Current or former smokers were defined ashaving had one year or more of tobacco use. Diabetes mellitus was defined as the presence of an active treatment with insulin or an oral antidiabetic agent; for patients administered dietary treatment, documentation of abnormal fasting blood glucose or glucose tolerance test based on the World Health Organization criteria was required for establishing this diagnosis.

All patients and control subjects were Chinese. They were all unrelated. Written informed consent was obtained from all subjects. The study protocol was approved by the Medical Ethics Committee of Jinhua.

\section{Genotyping of theTGF-beta1 Gene}

Venous blood $(5 \mathrm{ml})$ was collected into tubes containing EDTA (disodium salt, $50 \mathrm{mmol} / \mathrm{L}$ ), and genomic DNA was isolated with a DNA extraction kit (Roche, Switzerland). Genotyping of the codon 10 polymorphism of TGF-beta1 was performed by the allele-specific polymerase chain reaction (ASPCR) method. ${ }^{13}$ All reagents for PCR were purchased from Roche Diagnostics (Roche, Switzerland). The primer synthesized at the SBS Genetech Co (Beijing, China). The primer sequence was listed as follows: the generic primer (sense), 5' - gcccatctaggttatttccgtgggatactgagacac-3'; the $\mathrm{C}$ allele specific primer (antisense), 5'- cagcaccagtagccacagcagcg gtagcagcagcg-3'; the $\mathrm{T}$ allele specific primer (antisense), 5'tcagcaccagtagccacagcagcggtagcagcagca-3'. The PCR was performed with a reaction volume of $25 \mu \mathrm{L}$ containing $1 \mu \mathrm{L}$ of DNA, $1 \mu \mathrm{L}$ specific primer mix (consisting of generic primer and one of the two allele-specific primers), 12.5 $\mu \mathrm{L}$ Master-mix, and $9.5 \mu \mathrm{L} \mathrm{dd}_{2} \mathrm{O}$. Polymerase chain reaction amplification conditions were an initial denaturation at $95^{\circ} \mathrm{C}$ for five minutes, followed by 30 cycles of melting at $95^{\circ} \mathrm{C}$ for 30 s, annealing at $61^{\circ} \mathrm{C}$ for $45 \mathrm{~s}$, and extension at $72^{\circ} \mathrm{C}$ for $30 \mathrm{~s}$ with final extension at $72^{\circ} \mathrm{C}$ for five minutes.

The TGF-beta1 genotype was determined by $2.0 \%$ agarosegel electrophoresis of PCR products followed by ethidium bromide staining. The wild-type allele produced a 274-bp fragment, whereas the mutant allele produced a 273-bp fragment.

The DNA sequencing was performed on 20 random samples. It confirmed the match between the product sequence and the established TGF-beta1 sequence.

\section{Leukoaraiosis Grading and SVD Subtyping}

We obtained axial T1-, T2-, and proton density-weighted scans on 1.5-T Magnetic Resonance Imaging (MRI) scanners (GE Singna TwinSpeed) using 5-mm slice thickness. Slice thicknesses for CT scans varied from 5 to $8 \mathrm{~mm}$. The definitions leukoaraiosis and lacunes infarct were according to the previously described method ${ }^{2}$. Leukoaraiosis was defined as illdefined hyperintensities $\geq 5 \mathrm{~mm}$ on both $\mathrm{T} 2$ and proton density/ fluid-attenuated inversion recovery (FLAIR) MRI images without prominent hypointensities on T1-weighted MRI scans and as ill-defined and moderately hypodense areas of $\geq 5 \mathrm{~mm}$ on CT. Lacunes infarct was defined as well-defined hypodense areas of $>2 \mathrm{~mm}$ and $<15 \mathrm{~mm}$ on CT and as hyperintensities $>2 \mathrm{~mm}$ and $<15 \mathrm{~mm}$ on both $\mathrm{T} 2$ and proton density with hypointensities on T1-weighted and FLAIR MRI images. Periventricular low attenuation changes were rated on CT using the same scale in patients which were not available on MRI scans.

Leukoaraiosis on CT or MRI was evaluated blind to clinical and laboratory data by two independent radiologists using a semiquantitative rating scale,${ }^{14}$ modified to separate degrees of confluent leukoaraiosis as previously described ${ }^{2}$ : grade 0 , no leukoaraiosis; grade 1, mild leukoaraiosis (more than five white matter hyperintensities); grade 2, moderate confluent leukoaraiosis; and grade 3, severe confluent leukoaraiosis. In the event of a disagreement between the two radiologists the scans were arbitrated by a third assessor. In addition, on the basis of this leukoaraiosis grade, SVD patients were subtyped into two groups: isolated lacunar infarction (lacunar infarction with absent or mild leukoaraiosis), or ischemic leukoaraiosis (lacunar infarction in the presence of confluent leukoaraiosis) according to a previously validated method. ${ }^{2}$

\section{Statistical Analysis}

Power and Sample Size Calculation Software 2.1 was used for sample size calculation. We assumed $\alpha=0.05$ (two-sided) with power $=80 \%$ using a $1: 1$ ratio of cases to controls while 
looking for an odds ratio (OR) of 1.5. We assumed the rate of TT genotype in the source population (controls) was $30 \%$.

SPSS statistical software version 10.0 was used for statistical analysis. Data are presented as the mean \pm SD. Statistical significance was tested using unpaired Student's t-test or the Mann-Whitney U test as appropriate. Qualitative data were compared by the chi-square test. Allele frequencies were estimated by the gene-counting method, and chi-square test was performed to test for deviations from Hardy-Weinberg equilibrium. We also performed multivariable logistic regression analysis (backward: Wald elimination method) to adjust risk factors, with the type of subjects $(1=\mathrm{SVD}, 0=$ Controls $)$ as a dependent variable and independent variables including age, BMI, smoking status $(0=$ nonsmoker, $1=$ smoker $)$, metabolic variables $(0=$ no history of hypertension, diabetes mellitus, or hyperlipemia; 1=positive history), TGF-beta1 genotype and T allele $(\mathrm{T}=1, \mathrm{C}=0)$. TGF-beta1 genotype was calculated according to a dominant $(\mathrm{CC}=0, \mathrm{TC}=\mathrm{TT}=1)$ or recessive $(\mathrm{TC}=\mathrm{CC}=0$, $\mathrm{TT}=1$ ) genetic model. The OR and $95 \% \mathrm{CI}$ were also calculated. Statistical significance was taken as $P<0.05$. (2 tailed)

\section{RESULTS}

Three hundred and seventy seven patients $(85.49 \%)$ had MRI and $64(14.51 \%)$ had CT. A total of 112 patients were classified in the isolated lacunar infarction group had ( 98 by MRI). Fortyeight $(42.86 \%)$ patients in this group had lacunar infarction with absent leukoaraiosis whereas the other 64 (57.14\%) was lacunar infarction with mild leukoaraiosis. The 329 participants were classified in the ischaemic leukoaraiosis group (279 by MRI) of whom 143(43.46\%) had moderate leukoaraiosis and 186 $(56.53 \%)$ had severe leukoaraiosis.

The characteristics of the patients with cerebral SVD and controls are shown in Table 1 . There were no significant differences in age, BMI or conventional risk factors $(\mathrm{P}>0.05$;
Table 1). We also found that the prevalence of hypertension was more frequent among SVD patients than the control group $(\mathrm{P}<0.05$, Table 1). The same results were found when the prevalence of hypertension was compared in the two different SVD subtypes with control group $(\mathrm{P}<0.05$, Table 1). Differences between SVD subtypes with regard to clinical characteristics and risk factors were examined. We found the group with ischaemic leukoaraiosis had a higher frequency of diabetets $(\mathrm{P}<0.05$, Table 1$)$, but did not differ from the group with isolated lacunar infarction in other risk factors.

The genotype distributions among patients and controls were in Hardy-Weinberg equilibrium $(\mathrm{P}>0.05$. Subjects carrying TT genotype were more susceptible to $\mathrm{SVD}(\mathrm{OR}=1.42,95 \% \mathrm{CI}$, 1.06-1.90; $\mathrm{P}=0.019)$. When the SVD subtypes were investigated, TT genotype in a recessive model and $\mathrm{T}$ allele were significantly associated with ischaemic leukoaraiosis $(\mathrm{OR}=1.57,95 \mathrm{CI} \%$, 1.15-2.16, $\mathrm{P}=0.004 ; \mathrm{OR}=1.30,95 \% \mathrm{CI}, 1.06-1.60, \mathrm{P}=0.009)$ but not with lacunar infarction $(\mathrm{P}>0.05$; Table 2$)$.

On multivariate analysis controlling for age, smoking habit, and conventional vascular risk factors, the TT genotype was identified independently to be related to SVD (adjusted $\mathrm{OR}=1.438$, 95\%CI, 1.045-1.980; $\mathrm{P}=0.026)$. In analyzing the subtypes of SVD, the association of the TT genotype with ischaemic leukoaraiosis remained significant in a recessive model (adjusted $\mathrm{OR}=1.60,95 \% \mathrm{CI}, 1.14-2.45 ; \mathrm{P}=0.007$ ). Nevertheless, no significant association was found between $\mathrm{T}$ allele and ischaemic leukoaraiosis phenotype. In contrast, the significant association between $\mathrm{T}$ allele and ischaemic leukoaraiosis disappeared when adjusted for confounding.

\section{Discussion}

This study indicates subjects carrying TT genotype of TGFbeta1 codon 10 are independently associated with cerebral SVD, confined to only the group with leukoaraiosis.

Table 1: Baseline characteristics of the study population

\begin{tabular}{lcccc}
\hline Characteristic & Ctrls & SVD & Ischaemic leukoaraiosis & Isolated lacunar infarction \\
\hline & 450 & 441 & 329 & 112 \\
Age, y & $62.22 \pm 14.58$ & $63.71 \pm 15.2$ & $64.14 \pm 14.96$ & $62.45 \pm 16.04$ \\
Male, n (\%) & $326(72.44)$ & $313(70.97)$ & $227(68.99)$ & $86(76.78)$ \\
BMI,kg/m & $24.94 \pm 3.36$ & $24.57 \pm 4.56$ & $24.73 \pm 4.60$ & $24.06 \pm 4.44$ \\
Hyperlipemia,n (\%) & $96(21.33)$ & $91(20.63)$ & $74(22.49)$ & $17(15.17)$ \\
Smoking, n (\%) & $179(39.77)$ & $185(41.95)$ & $132(40.12)$ & $53(47.32)$ \\
Hypertension, n (\%) & $219(48.66)$ & $261(59.18)^{*}$ & $193(58.66) *$ & $68(60.71)$ \\
Diabetes, n (\%) & $75(16.7)$ & $82(18.6)$ & $71(21.6) \#$ & $11(9.8)$ \\
\hline
\end{tabular}

* $\mathrm{P}<0.01$ vs controls; $\mid \mathrm{P}<0.05$ vs controls; \# $\mathrm{P}<0.01$ vs lacunar infarction, $\mathrm{y}=$ years, $\mathrm{n}=$ number, $\mathrm{BMI}=$ body mass index 


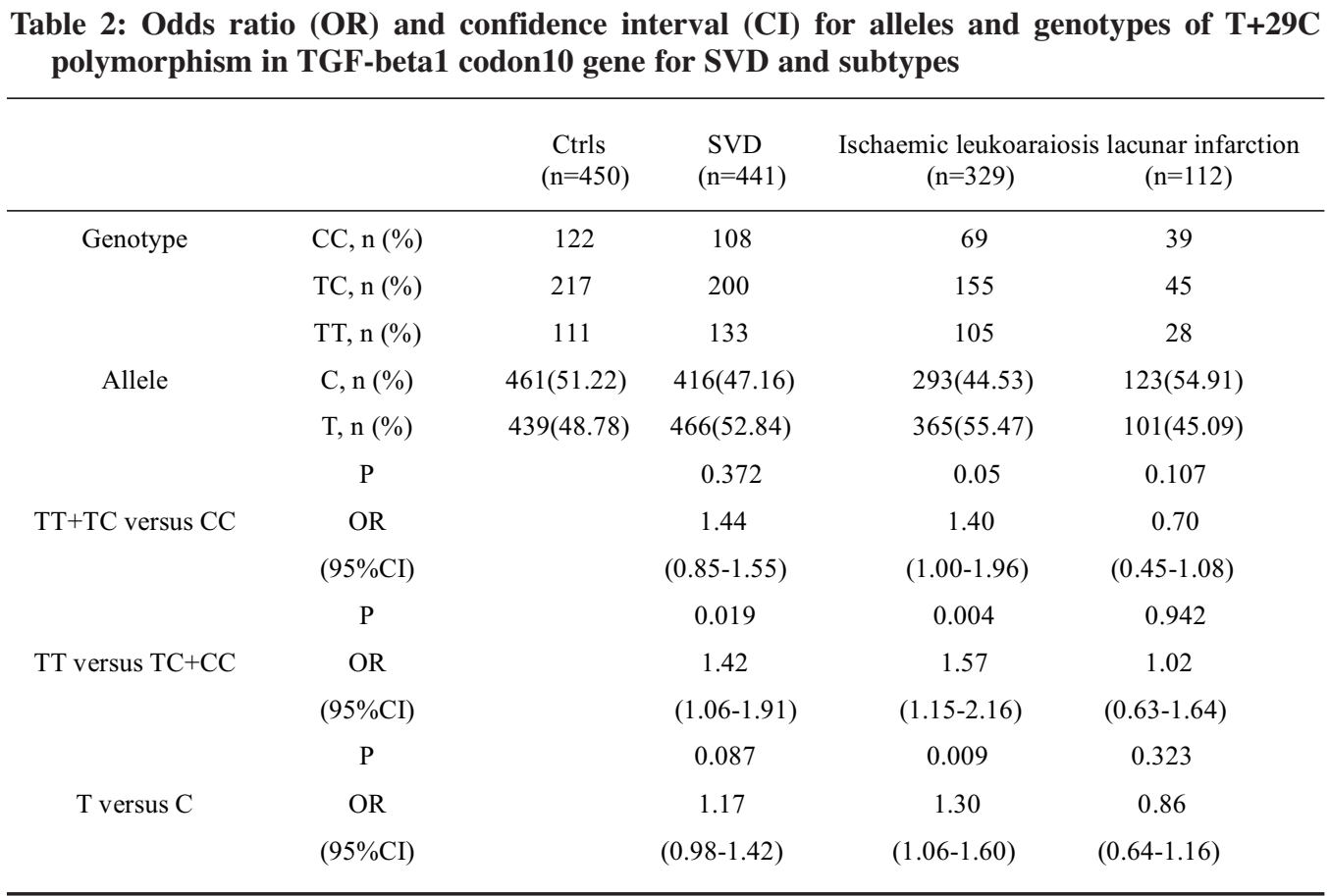

Two previous studies examined the $\mathrm{T}+29 \mathrm{C}$ variant as a risk factor in stroke. ${ }^{15,16}$ The sparse results available are inconsistent. In the large prospective cohort study ${ }^{15}$ with more than 6000 Caucasians individuals, $\mathrm{C}$ allele was found to be a risk factor for stroke as a whole but not for ischemic stroke. No further investigation in ischemic stroke subtypes was tested. Conversely, a small number of patients with lacunar stroke $(n=110)$ were studied as part of a larger investigation of genetic risk factors in all ischemic stroke phenotypes and a positive association was found between the TT genotype and the risk of both ischemic stroke and lacunar stroke $(\mathrm{OR}=1.63, \mathrm{P}=0.026 ; \mathrm{OR}=2.07$, $\mathrm{P}=0.006)$ in a Korean population ${ }^{16}$, thus leading potentially to spurious associations. To our knowledge, our study is the first one to examine the relationship between codon 10 polymorphisms of TGF-beta1gene and cerebral SVD subtypes. These results in our present study are in agreement with study by Kim et $\mathrm{al}^{16}$ and provide more convincing evidence of a causal relationship between TGF-beta1 and SVD because of controlling confounding variables and large numbers $(n=441)$ of well phenotyped subjects with cerebral SVD. Consequently, the present study may provide more insight into the pathogenesis of cerebral SVD and difference between phenotypes.

It has been well documented that TGF-beta1 is a pleiotropic cytokine with potent anti-inflammation property. Therefore, it is conceivable that TT genotype of $\mathrm{T}+29 \mathrm{C}$ polymorphism might be a risk for SVD, because the TT genotype is associated with a decreased TGF-beta1 level. ${ }^{10,11}$ Interestingly, in our study, the effect of TT genotype was confined to only the group with leukoaraiosis. It is suggested that different mechanisms are involved in the pathogenesis of the two subtypes, with microatheroma playing a major role in isolated lacunar infarcts and a diffuse arteriosclerosis being more important in ischemic leukoaraiosis. Consistent with this heterogeneity, we found differing associations between both the TGF-beta1 polymorphism and between the two subtypes of SVD. The frequency of TT genotype was highest in the ischaemic leukoaraiosis group. Therefore TGF-betal could be more important in the development of small vessel arteriosclerosis than microatheroma. The precise molecular genetic mechanism of SVD subtypes remains to be elucidated in further studies.

Our study has a number of strengths. The first is the accurate phenotyping of cerebral SVD and genotyping of TGF-beta1 codon 10. In this study, we used a carefully phenotyped population of SVD patients and excluded individuals with any other possible causes of stroke. Accurate classification of cerebral SVD was based on the use of advanced magnetic resonance imaging such as FLAIR $(n=304)$.

The main strength of our study includes FLAIR, which is reported to be more sensitive for detecting periventricular lesions than T2 sequences, and can effectively differentiate small ischaemic lesions from dilated perivascular spaces in the deep white matter ${ }^{17}$. A second is the homogenous genetically and ethnically study population and the appropriate control group, thus selection bias attributable to population stratification was avoided. Our study population resided in Jinhua and adjacent cities in the central part of Zhejiang province and the distribution of TGF-beta1 genotypes in our control group was also found in Hardy-Weinberg equilibrium, which strongly suggests that our study population was genetically homogeneous. 
The potential limitations of our study warrant consideration. Firstly, it still could not completely be ruled out that some of our controls did have asymptomatic cerebral SVD but this would not alter our conclusions, although control individuals were all examined using CT or MRI scans if the initial diagnosis was unclear to exclude SVD. Secondly, the effect of TGF-beta1 gene on serum TGF-beta1 level was not investigated in this study.

\section{CONCLUSION}

Our findings have provided evidence for the association of the $\mathrm{T}+29 \mathrm{C}$ polymorphism of the TGF-beta1 codon10 with leukoaraiosis subtype and indicated that TGF-beta1 play a role in the pathogenesis of diffuse leukoaraiosis. The potential mechanism of TGF-beta1 in the pathogenesis of SVD warrants further study.

\section{SOURCE OF FUNDING}

This study was supported by a grant of the Jinhua Science and Technology Bureau project, Zhejiang Province, The People's Republic of China (No: 2008-3-055).

\section{REFERENCES}

1. Erkinjuntti T, Kurz A, Gauthier S, Bullock R, Lillenfield S, Damaraju CV. Efficacy of galantamine in probable vascular dementia and Alzheimer's disease combined with cerebrovascular disease: a randomised trial. Lancet. 2002; 359: 1283-90.

2. Hassan A, Hunt BJ, O'Sullivan M, et al. Markers of endothelial dysfunction in lacunar infarction and ischaemic leukoaraiosis. Brain. 2003; 126: 424-32.

3. Boiten J, Lodder J, Kessel F. Two clinically distinct lacunar infarct entities? A hypothesis. Stroke. 1993; 24: 652-6.

4. Tomimoto H, Akiguchi I, Wakita H, Osaki A, Hayashi M, Yamamoto Y. Coagulation activation in patients with Binswanger disease. Arch Neurol. 1999; 56: 1104-8.

5. Khan U, Porteous L, Hassan A, Markus H. Risk factor profile of cerebral small vessel disease and its subtypes. J Neurol Neurosurg Psychiatry. 2007; 78: 702-6.

6. Gouw AA, Seewann A, van der Flier WM, et al. Heterogeneity of small vessel disease: a systematic review of MRI and histopathology correlations. J Neurol Neurosurg Psychiatry. 2011; 82: 126-35.

7. Di Napoli M, Papa F. C-reactive protein and cerebral small-vessel disease an opportunity to reassess small-vessel disease physiopathology? Circulation. 2005; 112: 781-5.

8. Fornage M, Chiang YA, O'Meara ES, et al. Biomarkers of inflammation and MRI-defined small vessel disease of the brain: the Cardiovascular Health Study. Stroke. 2008; 39: 1952-9.

9. Annes JP, Munger JS, Rifkin DB. Making sense of latent TGF beta activation. J Cell Sci. 2003; 116: 217-24.

10. Grainger DJ, Heathcote K, Chiano M, et al. Genetic control of the circulating concentration of transforming growth factor type beta1. Hum Mol Genet. 1999; 8: 93-7.

11. Yokota M, Ichihara S, Lin TL, Nakashima N, Yamada Y. Association of a T29-->C polymorphism of the transforming growth factor-betal gene with genetic susceptibility to myocardial infarction in Japanese. Circulation. 2000; 101: 2783-7.

12. Investigation committee of guideline for diagnosis and treatment of hyperlipemias, Japan Atherosclerosis Society. Guideline for diagnosis and treatment of hyperlipemias in adults. J Jpn Atheroscler Soc. 1997; 25: 1-34.

13. Newton CR, Graham A, Heptinstall LE, et al. Analysis of any point mutation in DNA. The amplification refractory mutation system (ARMS). Nucleic Acid Res. 1989; 17: 2503-16.

14. Fazekas F, Kleinert R, Offenbacher H, et al. Pathologic correlates of incidental MRI white matter signal hyperintensities. Neurology. 1993; 43: 1683-9.

15. Sie MP, Uitterlinden AG, Bos MJ, et al. TGF-beta 1 polymorphisms and risk of myocardial infarction and stroke: the Rotterdam Study. Stroke. 2006; 37: 2667-71.

16. Kim Y, Lee $\mathrm{C}$. The gene encoding transforming growth factor beta 1 confers risk of ischemic stroke and vascular dementia. Stroke. 2006; 37: 2843-5.

17. Herskovits EH, Itoh R, Melhem ER. Accuracy for detection of simulated lesions: comparison of fluid-attenuated inversionrecovery, proton density - weighted, and T2-weighted synthetic brain MR imaging. Am J Roentgenol. 2001; 176: 1313-18. 